\title{
Why anesthesiologists need to care about the way chronic pain is managed
}

\author{
Alexander J. Clark, MD • Christopher C. Spanswick, MBChB
}

Received: 1 May 2013/Accepted: 22 October 2013/Published online: 6 November 2013

(C) Canadian Anesthesiologists' Society 2013

Anesthesiologists are now frequently considered specialists in perioperative medicine. This view reflects changes in practice that have evolved over the last 20 years and are currently exemplified in many departments, including those specializing in pain medicine, pain management, and perioperative medicine. Postoperatively, many patients continue to receive inadequate analgesia, and poor pain control is the leading cause of post-discharge visits to the emergency room and early re-admission to hospital after surgery. ${ }^{1}$ Patients with preoperative chronic pain, especially those receiving opioids, have a high rate of unsatisfactory perioperative pain control. ${ }^{2}$

Chronic pain in Canada affects more than one in five Canadians. ${ }^{3}$ Consequently, many patients who come under our care suffer from chronic pain. These patients are more likely to be opioid-dependent and receiving one or more medications that act on the central nervous system. Patients' complex medication profiles and their concurrent anxiety and mood disruption can have a substantial impact on the perioperative care that the anesthesiologist provides. Herein, we consider some of the special issues that anesthesiologists should understand concerning the management of patients with chronic pain in the perioperative setting.

\footnotetext{
A. J. Clark, MD ( $\bowtie)$

Department of Anesthesia, Pain Management and Perioperative Medicine, Dalhousie University, Halifax, NS, Canada

e-mail: john.clark@cdha.nshealth.ca

C. C. Spanswick, MBChB

Department of Anesthesia, University of Calgary, Calgary, AB, Canada
}

\section{Preoperative pain, anxiety, and catastrophizing}

Preoperative anxiety and catastrophizing (i.e., a construct that includes fearing the worst, rumination, and magnification of symptoms- in this case pain) predict acute postoperative pain, and there is evidence that both may play a role in the development of chronic postsurgical pain. ${ }^{4,5}$ In addition, both factors have been implicated as predictors of analgesic use in the postoperative period. Catastrophizing also predicts poor quality of life after surgery. ${ }^{5}$ Preoperative anxiety and pain sensitivity have been shown to be independent predictors of propofol and sevoflurane requirements in general anesthesia as well as risk factors for poor pain control in the postoperative period. $^{6}$

The most important predictor of postoperative pain in ambulatory surgery is the presence of preoperative pain. Other factors that may also impact anesthesiologists' care include the anticipated degree of postoperative pain and the patient's high preoperative expectations regarding postoperative analgesia. ${ }^{7}$ Patients with preoperative pain, both related and unrelated to the surgical site, are at significant risk of developing chronic pain at the site of surgery. The longer the duration and higher the severity of the preexisting pain, the more likely that chronic postsurgical pain will develop. The rates of chronic postsurgical pain vary, for example, $30-50 \%$ of patients experience chronic pain following amputation, 20-30\% following breast surgery, 30-40\% following thoracotomy, and $10 \%$ following inguinal hernia repair. ${ }^{8}$ Patients with chronic pain report poor mental health-related quality of life and more severe psychosomatic dysfunction before surgery and three months after surgery. ${ }^{9}$

A recent review clearly indicates that preoperative pain, anxiety, age, and type of surgery are four significant 
predictors of postoperative pain. ${ }^{10}$ By being aware of these factors and taking them into account, the anesthesiologist may have an impact not only on pain in the immediate postoperative period but also on the longer term recovery.

\section{The opioid-tolerant patient with chronic pain}

The opioid-tolerant patient requiring acute pain management in the perioperative period presents the anesthesiologist with considerable challenges. Treatment goals should be directed toward providing relief from acute postsurgical pain, preventing opioid withdrawal, and continuing appropriate preoperative opioid strategies through to discharge. The patient's regular opioid medication must continue in the short term; however, to provide appropriate postoperative analgesia, there is often a need to increase the doses of opioid medication substantially and incorporate a prescription for non-opioid and adjuvant medication. In addition to managing opioid tolerance, there is always a possibility of opioid-induced hyperalgesia ${ }^{11}$ requiring additional pharmacological intervention.

Discharge planning should commence before the time of hospital admission, and both the anesthesiologist and the patient should have clear expectations for a limited duration of additional opioid augmentation and a welldefined plan for a reduction in opioid medication soon after the acute phase of recovery. ${ }^{12}$ Communication with the patient's primary care physician/continuing care physician is of paramount importance to ensure uninterrupted quality of care.

Opioid-tolerant patients have much higher opioid requirements post-surgery. For example, patients undergoing total knee arthroplasty and taking $\geq 30 \mathrm{mg}$ of morphine equivalents $p o$ per day receive up to five to six times the usual dose of opioids while in the postanesthesia care unit (PACU) and within the first $48 \mathrm{hr}$ after discharge from the PACU. ${ }^{2}$

Opioid-tolerant patients have higher pain scores than opioid-naive patients within the first $24 \mathrm{hr}$ of discharge from the PACU. ${ }^{2}$ The patient's usual dose of opioid medication should be administered with appropriate conversions from oral to intravenous equivalents (if necessary), and additional opioid requirements should be anticipated and provided to address the additional pain of the surgical procedure.

It has been suggested that patient-controlled analgesia (PCA) with higher bolus doses of opioids and shorter lockout intervals should be used in the opioid-tolerant patient. ${ }^{13}$ Nevertheless, use of PCA can lead to very challenging situations in the patient who has previously shown loss of control over their opioid medication. In this situation, a PCA needs to be used with care. In the authors' experience, a time-contingent mode of administration of opioid medication is often best, with frequent reassessment and clear communication of the expected duration of opioid dose and use.

The perioperative anesthesiologist needs to understand the distinction between addiction, dependence, and tolerance as it relates to the use of opioids. It is wise to consult with an addiction specialist, especially if the patient is taking methadone or buprenorphine for addiction prior to surgery. For those individuals addicted to opioids, the perioperative period is not a time to initiate weaning. ${ }^{14}$ The ANZAC Acute Pain Management: Scientific Evidence document provides a valuable management plan (http://www. anzca.edu.au/resources/college-publications/pdfs/Acute $\%$ 20Pain\%20Management/books-and-publications/acutepain. pdf; (accessed June $11^{\text {th }}, 2013$ ).

\section{The role of multimodal analgesia}

Perioperative multimodal analgesia, defined as the use of different classes of analgesics and different sites of analgesic administration, has been shown to enhance recovery, reduce hospital stay, and facilitate early convalescence. ${ }^{15}$ Local and regional anesthesia, in particular, (e.g., local infiltration, peripheral nerve block, neuraxial administration, etc.) along with multimodal analgesics (e.g., nonsteroidal anti-inflammatory drugs [NSAIDS], opioids, central nervous system modulators, etc.) may reduce the likelihood of developing chronic pain.

It is unclear whether patients with preexisting chronic pain benefit from multimodal analgesia for their acute postoperative pain. For example, medications, such as ketamine, an N-methyl D-aspartate (NMDA) receptor antagonist, have been used in subanesthetic doses in the opioid-tolerant patient having a spinal fusion in order to block the processing of nociceptive input in chronic pain syndromes. The patients who received low-dose ketamine on induction of general anesthesia and for the following 24 hr did not show statistically significant pain reduction during the first postoperative day, either at rest or with physical therapy, or reduced use of hydromorphone for pain control. ${ }^{16}$

A recent meta-analysis shows that preemptive analgesia does have an effect on postoperative pain scores, supplemental analgesic consumption, and time to first analgesic consumption. Preemptive epidural analgesia probably has the greatest effect, while local anesthetics for wound infiltration and NSAID administration have a lesser effect. The evidence for preemptive NMDA antagonists is equivocal, and there is lack of evidence that preemptive opioid administration has any additional effect. ${ }^{17}$ 
Does the type of anesthesia matter?

Studies are lacking on the effect of inhalation or intravenous anesthesia on pain in patients with preoperative chronic pain; however, it has recently been shown that inhalation anesthesia with sevoflurane is associated with a higher incidence of chronic pain after breast cancer surgery when compared with propofol anesthesia; propofol anesthesia did not have a significant effect on either the severity or duration of chronic pain. ${ }^{18}$ The use of nitrous oxide has been shown to decrease the risk of chronic postsurgical pain after major noncardiac surgery, ${ }^{19}$ and total intravenous anesthesia with propofol and remifentanil may reduce the incidence of chronic postsurgical pain after thoracotomy when compared with inhalation anesthesia. ${ }^{20}$ This is an area of anesthesiology that requires further study.

In conclusion, it is vital to perform a comprehensive preoperative assessment of the patient with chronic pain scheduled for anesthesia and surgery. This assessment should include a review of the severity of the patient's pain, an assessment of anxiety, depression, and catastrophizing, and a thorough review of the concurrent analgesic regimen, including identification of the pharmacotherapeutic modalities being used. The opioiddependent patient presents particular challenges that require careful preoperative planning and pre- and postoperative management strategies to ensure a smooth perioperative journey.

The anesthesiologist should be instrumental in early recognition of patients with chronic pain and in identification of factors that may lead to suboptimal pain control in the perioperative period. Careful planning of the patient's care throughout this period can mitigate these factors.

\section{Pourquoi les anesthésiologistes doivent s'inquiéter de la façon dont la douleur chronique est prise en charge}

De nos jours, les anesthésiologistes sont fréquemment considérés comme des experts en médecine périopératoire. Cette image est le reflet de certains des changements apportés à la pratique au cours des 20 dernières années; ces changements sont actuellement visibles dans de nombreux départements, y compris ceux se spécialisant en médecine de la douleur, en prise en charge de la douleur et en médecine périopératoire. Après leur opération, bon nombre de patients reçoivent toujours une analgésie inadaptée, et un mauvais soulagement de la douleur est la cause principale de visites à l'urgence après le congé de l'hôpital et de réadmission précoce à l'hôpital après une chirurgie. ${ }^{1}$ Les patients atteints de douleur chronique préopératoire, particulièrement ceux recevant des opioïdes, affichent un taux élevé de soulagement insatisfaisant de la douleur périopératoire. ${ }^{2}$

$\mathrm{Au}$ Canada, la douleur chronique affecte plus d'un Canadien sur cinq. ${ }^{3}$ En d'autres termes, de nombreux patients dont nous devons prendre soin souffrent de douleur chronique. Ces patients courent un risque plus élevé de dépendance aux opioïdes et prennent probablement un médicament ou plus qui agit sur le système nerveux central. Les profils médicamenteux complexes de ces patients, tout comme l'anxiété et les sautes d'humeur concomitantes dont ils souffrent, peuvent avoir un impact considérable sur les soins périopératoires fournis par l'anesthésiologiste. Dans cet éditorial, nous nous intéressons à certaines des problématiques spécifiques dont les anesthésiologistes devraient être conscients en matière de prise en charge de patients souffrant de douleur chronique dans un contexte périopératoire.

\section{Douleur préopératoire, anxiété et catastrophisation}

L'anxiété préopératoire et la catastrophisation (c.-à-d. un construit incluant la crainte du pire, des ruminations et la magnification des symptômes - dans le cas présent la douleur) sont des prédicteurs de douleur postopératoire aiguë et certaines données probantes soutiennent que ces deux facteurs jouent un rôle dans l'apparition de la douleur chronique postchirurgicale. ${ }^{4,5}$ En outre, ces facteurs ont tous deux été impliqués en tant que prédicteurs de l'utilisation d'analgésiques en période postopératoire. La catastrophisation est également un prédicteur de qualité de vie médiocre après une chirurgie. ${ }^{5}$ Il a été démontré que l'anxiété préopératoire et la sensibilité à la douleur étaient des prédicteurs indépendants de besoins élevés en propofol et en sévoflurane durant l'anesthésie générale et constituaient des facteurs de risque de mauvais soulagement de la douleur en période postopératoire. ${ }^{6}$

Le prédicteur le plus important de douleur postopératoire en chirurgie ambulatoire est la présence de douleur préopératoire. Parmi les autres facteurs pouvant également influencer les soins des anesthésiologistes, citons le degré anticipé de douleur postopératoire et les attentes préopératoires élevées du patient concernant l'analgésie postopératoire. ${ }^{7}$ Les patients souffrant de douleur préopératoire, qu'elle soit liée ou non au site chirurgical, courent un risque élevé de manifester de la douleur chronique au site de la chirurgie. Plus la douleur préexistante dure depuis longtemps et plus elle est grave, plus il est probable qu'une douleur chronique 
postchirurgicale se manifeste. Les taux de douleur chronique postchirurgicale varient. Par exemple, 30-50\% des patients souffrent de douleur chronique après une amputation, 20-30\% après une chirurgie mammaire, $30-40 \%$ après une thoracotomie et $10 \%$ après une cure d'hernie inguinale. $^{8}$ Les patients atteints de douleur chronique rapportent une qualité de vie médiocre liée à leur santé mentale ainsi qu'un dysfonctionnement psychosomatique plus grave avant la chirurgie et trois mois après la chirurgie. ${ }^{9}$

Un compte-rendu récent indique clairement que la douleur préopératoire, l'anxiété, l'âge et le type de chirurgie sont quatre prédicteurs significatifs de douleur postopératoire. ${ }^{10}$ En étant conscient de ces facteurs et en en tenant compte, l'anesthésiologiste peut avoir un impact non seulement sur la douleur en période postopératoire immédiate, mais également sur le rétablissement à long terme de son patient.

\section{Le patient tolérant aux opioïdes et souffrant de douleur chronique}

Quand il doit s'occuper d'un patient tolérant aux opiö̈des nécessitant une prise en charge de la douleur aiguë en période périopératoire, l'anesthésiologiste est confronté à des défis considérables. Les objectifs de traitement devraient inclure le soulagement de la douleur postchirurgicale aiguë, la prévention du sevrage des opioïdes et le maintien de stratégies préopératoires adaptées à base d'opioïdes durant tout le séjour et jusqu'au congé. À court terme, le patient doit continuer à recevoir ses médicaments opioïdes habituels; toutefois, afin de fournir une analgésie postopératoire adéquate, il faut souvent augmenter considérablement les doses et prescrire en plus des médicaments non opioïdes et des adjuvants. À la prise en charge de la tolérance aux opioïdes s'ajoute la possibilité d'une hyperalgésie induite par les opioïdes; ${ }^{11}$ une telle complication nécessitera alors une intervention pharmacologique supplémentaire.

La planification du congé devrait être faite avant même l'admission à l'hôpital, et l'anesthésiologiste et le patient devraient tous deux avoir des attentes claires quant à la durée limitée de l'augmentation des opioïdes ainsi qu'un plan bien défini de réduction rapide de ces médicaments après la phase aiguë du rétablissement. ${ }^{12}$ Il est essentiel de communiquer avec le médecin de soins primaires / de soins continus du patient afin de garantir une qualité de soins ininterrompue.

Les patients tolérants aux opioïdes ont des besoins en opiö̈des bien plus élevés après une chirurgie. Par exemple, les patients subissant une arthroplastie totale du genou et prenant $\geq 30 \mathrm{mg}$ d'équivalents morphine po par jour reçoivent jusqu'à cinq ou six fois la dose habituelle d'opioïdes pendant leur séjour en salle de réveil et au cours des 48 premières heures suivant leur congé de la salle de réveil. $^{2}$

Les patients tolérants aux opioïdes ont des scores de douleur plus élevés que les patients n'ayant jamais pris d'opioïdes au cours des 24 premières heures suivant le congé de la salle de réveil. ${ }^{2}$ La dose d'opioïdes habituelle du patient devrait être administrée en convertissant correctement la dose orale en son équivalent intraveineux (si nécessaire), et les besoins opioïdes supplémentaires devraient être anticipés et administrés afin de prendre en charge la douleur supplémentaire liée à l'intervention chirurgicale.

Des études ont suggéré qu'une analgésie contrôlée par le patient (ACP) avec des bolus plus élevés d'opioïdes et des intervalles d'interdiction plus courts devrait être utilisée chez le patient tolérant aux opioïdes. ${ }^{13}$ Toutefois, le recours à l'ACP peut créer des situations très difficiles pour un patient qui aurait, par le passé, perdu le contrôle sur ses médicaments opioïdes. Dans un tel cas, il faut faire attention lors de l'utilisation d'une ACP. Dans notre expérience, un mode d'administration des opioïdes en fonction du temps est souvent la meilleure solution, surtout lorsqu'il est accompagné d'une réévaluation fréquente et d'une communication claire de la durée prévue de la dose et de l'utilisation de l'opioïde.

Il est important que l'anesthésiologiste périopératoire comprenne la distinction entre toxicomanie, dépendance et tolérance en ce qui touche à l'utilisation d'opioïdes. Il est prudent de consulter un spécialiste en toxicomanie, et ce, particulièrement si le patient prend de la méthadone ou de la buprénorphine pour traiter une toxicomanie présente avant la chirurgie. Pour les personnes dépendantes aux opioïdes, la période périopératoire n'est pas le bon moment pour entreprendre un sevrage. ${ }^{14}$ Le document de l'ANZAC intitulé Acute Pain Management: Scientific Evidence (Prise en charge de la douleur aiguë : données probantes scientifiques) propose un excellent plan de prise en charge (http://www.anzca.edu.au/resources/college-publications/pdfs/ Acute\%20Pain\%20Management/books-and-publications/ acutepain.pdf; consulté le 11 juin 2013).

\section{Le rôle de l'analgésie multimodale}

Il a été démontré que l'analgésie périopératoire multimodale, définie en tant que l'utilisation de différentes classes d'analgésiques administrés à différents sites, améliorait le rétablissement, réduisait la durée de séjour à l'hôpital et facilitait une convalescence précoce. ${ }^{15}$ L'anesthésie locorégionale, en particulier (par ex., infiltration locale, bloc du nerf périphérique, 
administration neuraxiale, etc.), combinée à une analgésie multimodale (par ex., avec des anti-inflammatoires non stéroïdiens [AINS], des opioïdes, des modulateurs du système nerveux central, etc.) pourrait réduire la probabilité de souffrir de douleur chronique.

Nous ne savons pas si les patients souffrant de douleur chronique préexistante bénéficient d'une analgésie multimodale pour soulager leur douleur postopératoire aiguë. Par exemple certains médicaments, tels que la kétamine, un antagoniste des récepteurs du N-méthyl-D-aspartate (NMDA), ont été utilisés en doses sous-anesthésiques chez les patients tolérants aux opioïdes subissant une chirurgie du rachis afin de bloquer le traitement des informations nociceptives lors de syndromes de douleur chronique. Les patients ayant reçu une faible dose de kétamine à l'induction de l'anesthésie générale et durant les 24 heures suivantes n'ont pas montré de réduction statistiquement significative de la douleur durant le premier jour postopératoire, qu'ils soient au repos ou en traitement de physiothérapie, ou de réduction de leur utilisation d'hydromorphone pour soulager la douleur. ${ }^{16}$

Une méta-analyse récente a montré que l'analgésie préventive a un effet sur les scores de douleur postopératoires, la consommation d'analgésiques supplémentaires et le moment de première prise d'analgésique. L'analgésie péridurale préventive a probablement l'effet le plus prononcé, alors que les anesthésiques locaux par infiltration de plaie et l'administration d'AINS ont un effet moindre. Les données probantes appuyant l'utilisation d'antagonistes du NMDA sont équivoques, et nous manquons de données probantes étayant que l'administration préventive d'opioïdes ait un effet additionnel quelconque. ${ }^{17}$

\section{Est-ce que le type d'anesthésie est important?}

Les études sur l'effet de l'anesthésie par inhalation ou intraveineuse font défaut chez les patients souffrant de douleur chronique préopératoire; toutefois, on a récemment démontré que l'anesthésie par inhalation à base de sévoflurane était associée à une incidence plus élevée de douleur chronique après une chirurgie de cancer du sein comparativement à une anesthésie au propofol; l'anesthésie au propofol n'a pas eu d'effet significatif sur la gravité ou la durée de la douleur chronique. ${ }^{18}$ Il a été démontré que l'utilisation de protoxyde d'azote réduisait le risque de douleur chronique postchirurgicale après une chirurgie non cardiaque majeure, et l'anesthésie intraveineuse totale réalisée avec du propofol et du rémifentanil pourrait réduire l'incidence de douleur chronique postchirurgicale après une thoracotomie comparativement à une anesthésie par inhalation. ${ }^{20} \mathrm{Ce}$ domaine de l'anesthésiologie nécessite des études supplémentaires.

En conclusion, il est essentiel de réaliser une évaluation préopératoire complète du patient atteint de douleur chronique devant subir une anesthésie et une chirurgie. Cette évaluation doit passer en revue la gravité de la douleur du patient, évaluer son anxiété, sa dépression et sa tendance à la catastrophisation, et inclure un examen exhaustif des analgésiques pris simultanément en identifiant bien les modalités pharmacothérapeutiques utilisées. Le patient dépendant aux opioïdes présente des défis particuliers qui nécessitent une planification préopératoire minutieuse ainsi que des stratégies de prise en charge pré- et postopératoires afin de garantir un cheminement périopératoire sans accroc.

L'anesthésiologiste doit jouer un rôle important pour reconnaitre tôt les patients souffrant de douleur chronique et identifier les facteurs qui pourraient donner lieu à un soulagement sous-optimal de la douleur en période périopératoire. Une planification minutieuse des soins du patient tout au long de cette période peut contribuer à réduire l'impact de ces facteurs.

Acknowledgements The authors sincerely thank Dr. Ian Beauprie for his invaluable comments on the manuscript.

Competing interests None declared.

Conflits d'intérêt Aucun.

\section{References}

1. Lynch $M E$. The need for a Canadian pain strategy. Pain Res Manag 2011; 16: 77-80.

2. Patanwala AE, Jarzyna DL, Miller MD, Erstad BL. Comparison of opioid requirements and analgesic response in opioid-tolerant versus opioid-naïve patients after total knee arthroplasty. Pharmacotherapy 2008; 28: 1453-60.

3. Boulanger A, Clark AJ, Squire P, Chui E, Horbay GL. Chronic pain in Canada: have we improved our management of chronic noncancer pain? Pain Res Manag 2007; 12: 39-47.

4. Theunissen M, Peters ML, Bruce J, Gramke HF, Marcus MA. Preoperative anxiety and catastrophizing: a systematic review and meta-analysis of the association with chronic postsurgical pain. Clin J Pain 2012; 28: 819-41.

5. Khan RS, Ahmed K, Blakeway E, et al. Catastrophizing: a predictive factor for postoperative pain. Am J Surg 2011; 201: 122-31.

6. Kil HK, Kim WO, Chung WY, Kim GH, Seo H, Hong JY. Preoperative anxiety and pain sensitivity are independent predictors of propofol and sevoflurane requirements in general anaesthesia. Br J Anaesth 2012; 108: 119-25.

7. Gramke HF, de Rijke JM, van Kleef $M$, et al. Predictive factors of postoperative pain after day-case surgery. Clin J Pain 2009; 25: 455-60.

8. Kehlet H, Jensen TS, Woolf CJ. Persistent postsurgical pain: risk factors and prevention. Lancet 2006; 367: 1618-25.

9. Gerbershagen HJ, Ozgur E, Dagtekin $O$, et al. Preoperative pain as a risk factor for chronic post-surgical pain - six month followup after radical prostatectomy. Eur J Pain 2009; 13: 1054-61. 
10. Ip HY, Abrishami A, Peng PW, Wong J, Chung F. Predictors of postoperative pain and analgesic consumption: a qualitative systematic review. Anesthesiology 2009; 111: 657-77.

11. Ballantyne JC, Shin NS. Efficacy of opioids for chronic pain: a review of the evidence. Clin J Pain 2008; 24: 469-78.

12. Huxtable CA, Roberts LJ, Somogyi AA, MacIntyre PE. Acute pain management in opioid-tolerant patients: a growing challenge. Anaesth Intensive Care 2011; 39: 804-23.

13. Mehta V, Langford RM. Acute pain management for opioid dependent patients. Anaesthesia 2006; 61: 269-76.

14. Richebe $P$, Beaulieu $P$. Perioperative pain management in the patient treated with opioids: continuing professional development. Can J Anesth 2009; 56: 969-81.

15. Joshi GP. Multimodal analgesia techniques and postoperative rehabilitation. Anesthesiol Clin North America 2005; 23: 185202.
16. Urban MK, Ya Deau JT, Wukovits B, Lipnitsky JY. Ketamine as an adjunct to postoperative pain management in opioid tolerant patients after spinal fusions: a prospective randomized trial. HSS J 2008; 4: 62-5.

17. Ong CK, Lirk P, Seymour RA, Jenkins BJ. The efficacy of preemptive analgesia for acute postoperative pain management: a meta-analysis. Anesth Analg 2005; 100: 757-73.

18. Cho AR, Kwon JY, Kim KH, et al. The effects of anesthetics on chronic pain after breast cancer surgery. Anesth Analg 2013; 116 : 685-93.

19. Chan MT, Wan AC, Gin T, Leslie K, Myles PS. Chronic postsurgical pain after nitrous oxide anesthesia. Pain 2011; 152: 2514-20.

20. Song JG, Shin JW, Lee EH, et al. Incidence of post-thoractomy pain: a comparison between total intravenous anaesthesia and inhalation anaesthesia. Eur J Cardiothorac Surg 2012; 41: 1078-82. 